\title{
$\mathrm{N}(\mathrm{N})$ LO event files: applications and prospects
}

\author{
Daniel Maître* \\ IPPP Durham \\ E-mail: daniel.maitreddurham.ac.uk

\section{Gudrun Heinrich} \\ Max Planck Institute for Physics \\ Föhringer Ring 6 \\ 80805 Munich, Germany \\ E-mail: gudrun@mpp.mpg • de \\ Mark Johnson \\ Manchester University and the Cockcroft Institute \\ E-mail: mark.andrew.johnson@cern.ch
}

In this contribution we comment on the use of NLO n-Tuples and consider their extension to NNLO. As an application of n-Tuples we present preliminary results of a strong coupling extraction from a measurement of the production of $Z+2,3,4$ jets at the $\mathrm{LHC}$ at $7 \mathrm{TeV}$.

Loops and Legs in Quantum Field Theory

24-29 April 2016

Leipzig, Germany

${ }^{*}$ Speaker. 


\section{1. n-Tuples}

The calculation of high multiplicity processes at NLO in now possible for a large number of processes in a largely automated way (see e.g. [1] for a review). While they can be automated, high multiplicity NLO calculations still require a large amount of CPU time to be performed with an acceptable statistical precision. The most expensive part of the calculation is the computation of the matrix elements. Other parts of the calculation such as the evaluation of the parton distribution functions, jet clustering and the construction of observables and differential cross sections to be histogrammed are typically much less demanding. In practice this also requires to evaluate very similar expressions, differing only by a factor in the factorisation or renormalisation scales or by the PDF used. To avoid recalculations of the same matrix element many times one can store the matrix elements, the kinematic information and the coefficients of logarithms involving the renormalisation and factorisation scales in a file and read them back in, instead of recalculating the whole matrix element. This strategy, using ROOT [2] as the storage back-end, has been described in [3].

Besides the computational efficiency, this strategy has the advantage of facilitating the dissemination of the NLO results. The clear disadvantage is that the event files tend to be very large. The advantages outweigh this disadvantage for NLO calculations and this strategy has been used to produce NLO predictions [4, 5, 6, 7, 8, 9] for many Standard Model measurements $[10,11,12,13,14,15,16]$.

It is natural to wonder whether a similar approach would facilitate the calculation and dissemination of NNLO calculations. The advantages and disadvantages are the same: the matrix elements are computationally expensive to calculate and would benefit from being stored but the amount of events needed would lead to large file sizes. A first study in ref. [1] investigated the size of NNLO event files using the EERAD 3 program [17].

One significant change encountered when going from NLO to NNLO is the increase in the number of subtraction terms, which leads to a large number of phase-space configurations that need to be stored in the event file. Instead of storing each set of mapped momenta, one can store the original momenta they are mapped from, along with a reference to the procedure with which the mapped momenta are arrived at. This saves about an order of magnitude of storage space, at the cost of delegating the calculation of the mapped momenta to the user of the event file. Figure 1 shows the storage sizes as a function of the number of phase-space points.

Based on the extrapolation in figure 1 the usage of NNLO event files appears feasible in this case. The study in [1] used a leptonic initial state. One can anticipate that processes with hadronic initial states will be more challenging and require higher statistics, as there are additional integrations over the initial momentum fraction of the partons. This can make the prospects of using event files less favourable. However, there are several reasons to think hadronic processes might still be tractable: a) so far NNLO calculations have been optimised for CPU time, one could optimise them with respect to storage space (using the mapping information is an example of such an optimisation), b) new subtraction techniques such as $q_{T}$-subtraction [18] and $n$-jettiness [19, 20, 21] offer an easier subtraction structure which is closer to that of two separate NLO calculations than "genuine" NNLO subtraction schemes [22, 23, 24]. This "NLO-like" structure should lead to more efficient storage possibilities. 


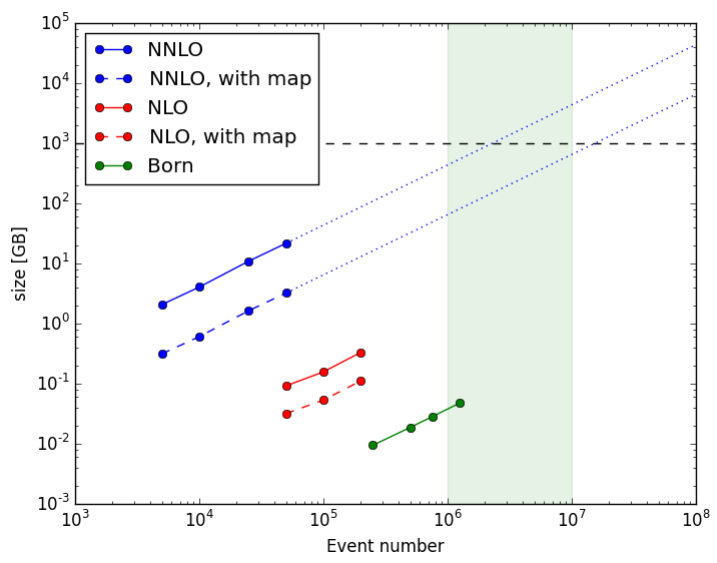

Figure 1: Event file size for $e^{+} e^{-} \rightarrow 3$ jets as a function of the number of events. The green band represents the number of points needed for a reasonable statistical error. The horizontal dashed line is the $1 \mathrm{~TB}$ threshold which corresponds to the order of magnitude of storage for NLO processes.

\section{Strong coupling determination}

The ability to recalculate the NLO predictions for a high multiplicity process relatively cheaply offers new opportunities for these processes to be used in phenomenological applications. We explore one of them in this section, namely extracting the value of the strong coupling constant from $Z+2,3,4$ jets measurements. The reason for using high multiplicity processes is that they display an increasingly strong dependence on $\alpha_{S}\left(M_{Z}\right)$ as the multiplicity increases, as illustrated in figure 2 .

For our $\alpha_{S}$ extraction we use the data from [25] for the rapidity and transverse momentum distribution of the $n$-th jet in $Z+n$ jets events. We compare it with predictions obtained from the BlackHat+Sherpa collaboration [4]. We used the n-Tuples they provide to generate a fast NLO grid [27] to speed up the calculation of the PDF covariance matrices and scale variations. The fit is obtained by minimising the $\chi^{2}$ function

$$
\chi^{2}\left(\alpha_{S}\right)=\left(y_{t}\left(\alpha_{s}\right)-y_{d}\right)^{T} C^{-1}\left(y_{t}\left(\alpha_{s}\right)-y_{d}\right),
$$

where $y_{t}$ is the theory prediction and $y_{d}$ are the experimental values. The covariance matrix $C$ is given by

$$
C=C_{\text {exp }}+C_{p d f}+C_{\text {theory }},
$$

with $C_{\exp }$ the experimental error covariance matrix, $C_{p d f}$ the PDF covariance matrix obtained using the LHAPDF library [28] and $C_{\text {theory }}$ the statistical covariance matrix of the theory prediction.

Figure 3 shows the preliminary results for the three PDF sets MSTW2 008 [29], CT10 [30] and NNPDF 2. 3 [31]. These sets were chosen to facilitate the comparison with the results obtained in ref. [25]. The results are given for fits to different sets of histograms: a) to the transverse momentum and rapidity of the $n$-th $p_{T}$-ordered jet for each multiplicity individually, b) for the combination of the transverse momentum and rapidity for each multiplicity, c) for the combination of all three 


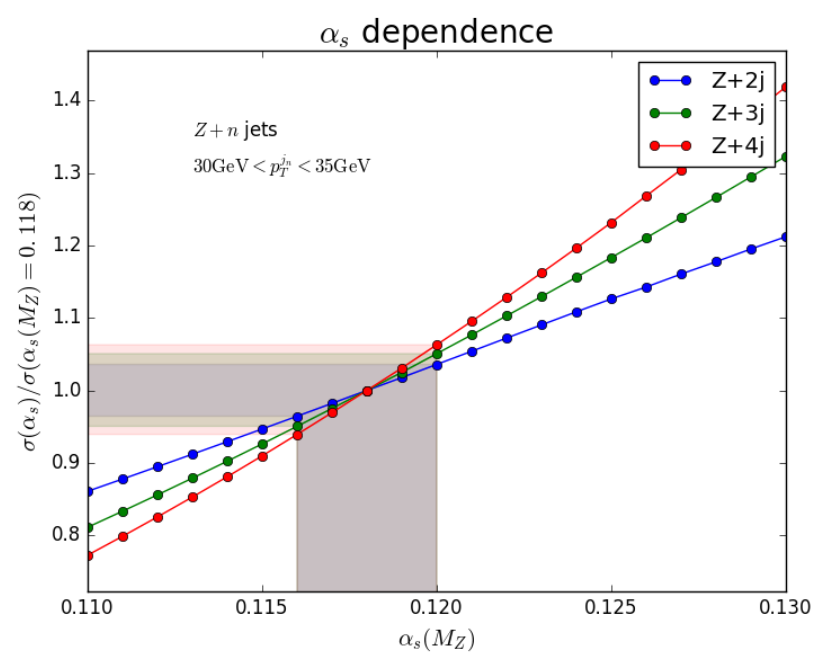

Figure 2: Left-hand panel: $\alpha_{S}\left(M_{Z}\right)$ dependence of the value of the $30 \mathrm{GeV}<p_{T}<35 \mathrm{GeV}$ bin in the transverse momentum distribution for the $n$-th jet for $n=2,3,4$.This figure illustrates the fact that a larger uncertainty on the cross section for a higher-multiplicity process can result in the same uncertainty on the predicted values of $\alpha_{S}\left(M_{Z}\right)$.

transverse momentum distributions, d) for the combination of all the rapidity distributions, e) for a combination of all histograms.

\section{Conclusion}

In this contribution we reported on an investigation of the suitability of the n-Tuple strategy for NNLO calculations. This strategy seems tractable for processes with no hadronic initial states and while the jury is still out for the case of hadronic initial states we have good reasons to think it will also be tractable in these cases. We also presented preliminary results for an extraction of the strong coupling constant from high multiplicity processes. The accuracy obtained for the value of $\alpha_{S}\left(M_{Z}\right)$ seems comparable with other determinations at the LHC.

\section{References}

[1] J. R. Andersen et al., Les Houches 2015: Physics at TeV Colliders Standard Model Working Group Report, in 9th Les Houches Workshop on Physics at TeV Colliders (PhysTeV 2015) Les Houches, France, June 1-19, 2015, 2016. 1605.04692.

[2] R. Brun and F. Rademakers, ROOT: An object-oriented data analysis framework, Nucl. Instrum. Meth. A389 (1997) 81-86.

[3] Z. Bern, L. Dixon, F. Febres Cordero, S. Höche, H. Ita, et al., Ntuples for NLO Events at Hadron Colliders, Comput.Phys.Commun. 185 (2014) 1443-1460, [1310. 7439 ].

[4] H. Ita, Z. Bern, L. J. Dixon, F. Febres Cordero, D. A. Kosower, and D. Maître, Precise predictions for $Z+4$ jets at hadron colliders, Phys. Rev. D85 (2012) 031501, [1108.2229]. 


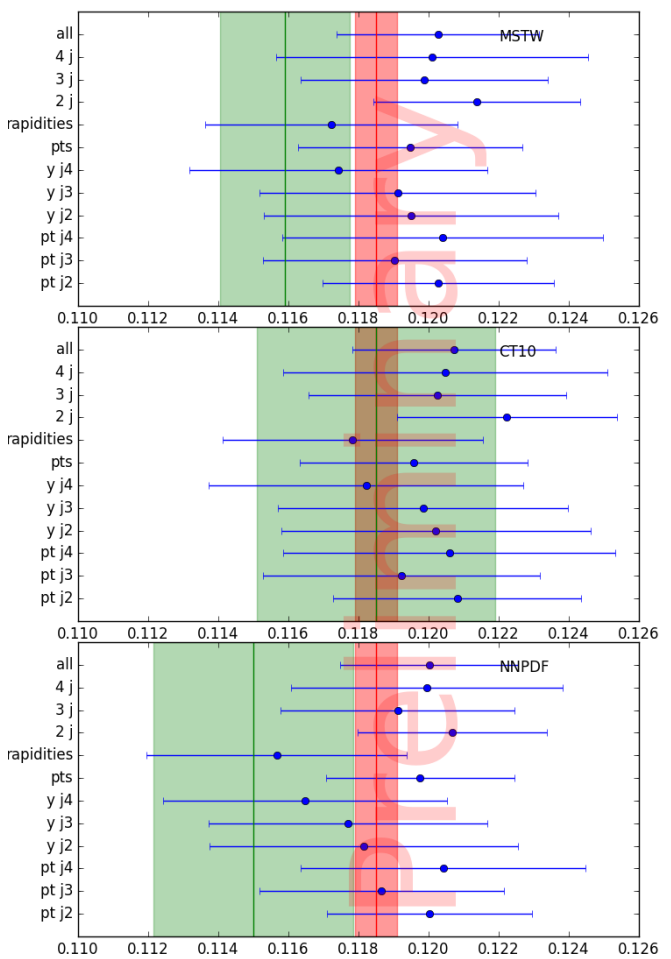

Figure 3: Strong coupling values for different PDF sets for each considered histogram and combinations. The green band is the result from [25]. The red band is the world average [26]. The boundaries of the blue error bars represent the values of $\alpha_{S}\left(M_{Z}\right)$ for which the $\chi^{2}-\chi_{\min }^{2}=1$. These error estimates do not include scale variation.

[5] C. Berger, Z. Bern, L. J. Dixon, F. Febres Cordero, D. Forde, T. Gleisberg, H. Ita, D. A. Kosower, and D. Maître, Precise predictions for W + 4-jet production at the Large Hadron Collider, Phys. Rev. Lett. 106 (2011) 092001, [1009.2338].

[6] Z. Bern, G. Diana, L. J. Dixon, F. Febres Cordero, S. Höche, H. Ita, D. A. Kosower, D. Maître, and K. Ozeren, Four-jet production at the Large Hadron Collider at next-to-leading order in QCD, Phys. Rev. Lett. 109 (2011) 042001, [1112.3940].

[7] N. Greiner, S. Höche, G. Luisoni, M. Schönherr, J.-C. Winter, and V. Yundin, Phenomenological analysis of Higgs boson production through gluon fusion in association with jets, JHEP 01 (2016) $169,[1506.01016]$.

[8] S. Badger, B. Biedermann, P. Uwer, and V. Yundin, Next-to-leading order QCD corrections to five jet production at the LHC, Phys. Rev. D89 (2014), no. 3 034019, [1309. 6585].

[9] S. Badger, A. Guffanti, and V. Yundin, Next-to-leading order QCD corrections to di-photon production in association with up to three jets at the Large Hadron Collider, JHEP 03 (2014) 122, [1312.5927].

[10] D0 Collaboration, V. M. Abazov et al., Studies of W boson plus jets production in p $\bar{p}$ collisions at $\sqrt{s}=1.96$ TeV, Phys. Rev. D88 (2013), no. 9 092001, [1302.6508]. 
[11] CMS Collaboration, V. Khachatryan et al., Differential cross section measurements for the production of a W boson in association with jets in protonâĂŞproton collisions at $\sqrt{s}=7$ TeV, Phys. Lett. B741 (2015) 12-37, [1406.7533].

[12] CMS Collaboration, V. Khachatryan et al., Measurements of jet multiplicity and differential production cross sections of $Z+$ jets events in proton-proton collisions at $\sqrt{s}=7$ TeV, Phys. Rev. D91 (2015), no. 5 052008, [1408.3104].

[13] ATLAS Collaboration, G. Aad et al., Measurement of four-jet differential cross sections in $\sqrt{s}=8$ TeV proton-proton collisions using the ATLAS detector, JHEP 12 (2015) 105, [1509. 07335 ].

[14] ATLAS Collaboration, G. Aad et al., A measurement of the ratio of the production cross sections for $W$ and $Z$ bosons in association with jets with the ATLAS detector, Eur. Phys. J. C74 (2014), no. 12 3168, [1408.6510].

[15] ATLAS Collaboration, G. Aad et al., Measurements of the W production cross sections in association with jets with the ATLAS detector, Eur. Phys. J. C75 (2015), no. 2 82, [1409. 8639 ].

[16] ATLAS Collaboration, G. Aad et al., Measurement of the production cross section of jets in association with a $Z$ boson in pp collisions at $\sqrt{s}=7$ TeV with the ATLAS detector, JHEP 07 (2013) 032, [1304.7098].

[17] A. Gehrmann-De Ridder, T. Gehrmann, E. W. N. Glover, and G. Heinrich, Infrared structure of e+e$\longrightarrow 3$ jets at NNLO, JHEP 11 (2007) 058, [0710.0346].

[18] S. Catani and M. Grazzini, An NNLO subtraction formalism in hadron collisions and its application to Higgs boson production at the LHC, Phys. Rev. Lett. 98 (2007) 222002, [hep-ph/ 0703012 ].

[19] I. W. Stewart, F. J. Tackmann, and W. J. Waalewijn, N-Jettiness: An Inclusive Event Shape to Veto Jets, Phys. Rev. Lett. 105 (2010) 092002, [1 004 . 2489].

[20] J. Gaunt, M. Stahlhofen, F. J. Tackmann, and J. R. Walsh, N-jettiness Subtractions for NNLO QCD Calculations, JHEP 09 (2015) 058, [1505.04794].

[21] R. Boughezal, C. Focke, X. Liu, and F. Petriello, W-boson production in association with a jet at next-to-next-to-leading order in perturbative QCD, Phys. Rev. Lett. 115 (2015), no. 6062002 , [1504.02131].

[22] A. Gehrmann-De Ridder, T. Gehrmann, and E. W. N. Glover, Antenna subtraction at NNLO, JHEP 09 (2005) 056, [hep-ph/0505111].

[23] J. Currie, E. W. N. Glover, and S. Wells, Infrared Structure at NNLO Using Antenna Subtraction, JHEP 04 (2013) 066, [1301.4693].

[24] M. Czakon, A novel subtraction scheme for double-real radiation at NNLO, Phys. Lett. $\mathbf{B 6 9 3}$ (2010) 259-268, [1 005 . 0274].

[25] CMS Collaboration, V. Khachatryan et al., Constraints on parton distribution functions and extraction of the strong coupling constant from the inclusive jet cross section in pp collisions at $\sqrt{s}=7 \mathrm{TeV}$, Eur. Phys. J. C75 (2015), no. 6 288, [1410.6765].

[26] Particle Data Group Collaboration, K. A. Olive et al., Review of Particle Physics, Chin. Phys. C38 (2014) 090001.

[27] fastNLO Collaboration, D. Britzger, K. Rabbertz, F. Stober, and M. Wobisch, New features in version 2 of the fastNLO project, in Proceedings, 20th International Workshop on Deep-Inelastic Scattering and Related Subjects (DIS 2012), pp. 217-221, 2012. 1208.3641. 
[28] M. R. Whalley, D. Bourilkov, and R. C. Group, The Les Houches Accord PDFs (LHAPDF) and Lhaglue, (2005) [hep-ph/0508110].

[29] A. Martin, W. Stirling, R. Thorne, and G. Watt, Parton distributions for the LHC, Eur. Phys. J. C63 (2009) 189-285, [0901.0002].

[30] J. Gao, M. Guzzi, J. Huston, H.-L. Lai, Z. Li, P. Nadolsky, J. Pumplin, D. Stump, and C. P. Yuan, CT10 next-to-next-to-leading order global analysis of QCD, Phys. Rev. D89 (2014), no. 3 033009, [1302.6246].

[31] R. D. Ball et al., Parton distributions with LHC data, Nucl. Phys. B867 (2013) 244-289, [1207.1303]. 